\title{
Exponential convergence of mixed hp-DGFEM for Stokes flow in polygons
}

\section{Journal Article}

\section{Author(s):}

Schötzau, Dominik; Wihler, Thomas P.

Publication date:

2003-12

Permanent link:

https://doi.org/10.3929/ethz-b-000052903

Rights / license:

In Copyright - Non-Commercial Use Permitted

Originally published in:

Numerische Mathematik 96(2), https://doi.org/10.1007/s00211-003-0478-5 


\section{Exponential convergence of mixed hp-DGFEM for Stokes flow in polygons}

\section{Dominik Schötzau ${ }^{1, \star}$, Thomas P. Wihler ${ }^{2}$}

1 Department of Mathematics, University of Basel, 4051 Basel, Switzerland; e-mail: schotzau@math.unibas.ch

2 Seminar for Applied Mathematics, ETH Zürich, 8092 Zürich, Switzerland; e-mail: twihler@sam.math.ethz.ch

Received September 10, 2002 / Revised version received March 7, 2003 /

Published online August 5, 2003 - (C) Springer-Verlag 2003

Summary. We analyze mixed $h p$-discontinuous Galerkin finite element methods (DGFEM) for Stokes flow in polygonal domains. In conjunction with geometrically refined quadrilateral meshes and linearly increasing approximation orders, we prove that the $h p$-DGFEM leads to exponential rates of convergence for piecewise analytic solutions exhibiting singularities near corners.

Mathematics Subject Classification (2000): 65N30

\section{Introduction}

Over the last few years, several mixed discontinuous Galerkin finite element methods (DGFEM) have been proposed for the discretization of incompressible fluid flow problems. We mention here only the piecewise solenoidal discontinuous Galerkin methods introduced in [5,25], the local discontinuous Galerkin methods of $[12,11]$, and the interior penalty methods studied in $[24,33,18]$. Some of the main motivations that led to the above methods are the following: First of all, the discontinuous nature of the finite element spaces allows one to easily treat convective terms by suitable upwind fluxes, similarly to the original discontinuous Galerkin discretizations of (non-linear) hyperbolic equations (see $[13,10,14]$ and the references therein). Thus, mixed DG methods provide robust and high-order accurate approximations

* The first author was partially supported by the Swiss National Science Foundation under Project 2100-068126.02

Correspondence to: D. Schötzau 
particularly in transport-dominated regimes; see, e.g., $[25,11,18]$ for mixed DGFEM for the Navier-Stokes and Oseen equations. Moreover, discontinuous Galerkin approaches are extremely flexible in the mesh-design; meshes with hanging nodes, elements of various types and shapes, and local spaces of different orders can be easily dealt with. Finally, mixed DG methods are remarkably flexible in the choice of velocity-pressure combinations, without extensive stabilization techniques. In the discontinuous Galerkin context, for example, no extra stabilization is needed to use optimal mixed-order elements where the approximation degree for the pressure is of one order lower than that of the velocity; see $[24,33]$ for details.

The recent work [29] presented a unifying framework for the analysis of mixed $h p$-DGFEM for pure Stokes flow. For $\mathcal{Q}^{k}-\mathcal{Q}^{k-1}$ elements, the dependence of the discrete inf-sup constant on the polynomial degree $k$ was shown to be of the order $\mathcal{O}(1 / k)$, for two- and three-dimensional domains. In three dimensions, this is exactly the same bound as that of [32] for conforming mixed $h p$-FEM, but with an optimal gap of one order in the finite element spaces for the velocity and the pressure. The results in [29] then ensure (slightly suboptimal) error bounds for the $p$-version of the DGFEM where convergence is obtained by increasing the polynomial approximation order on a fixed (quasi-uniform) mesh. However, these bounds give algebraic rates of convergence and are restricted to piecewise smooth solutions; an assumption that is unrealistic in domains with corners, due to the presence of corner singularities, see, e.g., $[15,26,23]$. For conforming mixed methods, similar $p$-version results can be found in, e.g., $[6,32,8,31,7]$ and the references cited therein.

In this paper, we extend the $h p$-approach of [29] to mixed $h p$-DGFEM for Stokes flow in polygonal domains where the exact solutions are piecewise analytic, but exhibit singularities at the corners. To describe the regularity of the exact solutions, we use a recent result from [23] that measures analytic regularity in terms of the countably normed, weighted spaces that were introduced by Babuška and Guo for closely related potential and elasticity problems; see $[20,21,19,2-4,22,30]$ and the references cited therein. The reduced regularity near corners imposes several technical difficulties and requires a careful treatment of the elements and the numerical fluxes at the vertices of the domain. By the use of new trace theorems for functions in weighted Sobolev spaces, we first show that the mixed $h p$-DGFEM is in fact well-defined. Then, we employ standard $h p$-version mesh design principles to resolve corner singularities: namely, we use meshes that are geometrically refined towards corners and approximation degrees that increase linearly away from corners. We show that this combination of $h$ - and $p$-refinement leads to exponential rates of convergence. For $h p$-DGFEM discretizations of scalar diffusion problems an analogous result was recently obtained in [35]. 
To prove exponential convergence for our mixed methods, we use several ingredients from the analysis of conforming mixed $h p$-FEM for Stokes flow on geometric meshes; see, e.g., [31,30,28], combined with the techniques that were developed in $[35,34]$ to treat diffusion and elasticity problems in polygons. Furthermore, we use the setting of [29] to derive the exponential convergence result. Exemplarily, we only consider the so-called interior penalty DGFEM, but point out that our results hold true verbatim for all the DG methods studied in [29]. We also note that our analysis can be straightforwardly extended to mixed formulations of linear elasticity problems with nearly incompressible materials; see, e.g., $[9,16]$.

The outline of the paper is as follows. In Section 1.1 we begin by introducing some notational conventions that we use throughout the paper. Section 2 reviews the analytic regularity of the Stokes problem in polygonal domains. In Section 3, we introduce meshes and establish several properties of functions on the elements near the corners of the domain. The $h p$-DGFEM discretization of the Stokes problem is introduced in Section 4. In Section 5, we derive abstract error estimates for piecewise analytic solutions. Section 6 is devoted to the main result of this paper; we prove that the $h p$-DGFEM is exponentially convergent. We end our presentation with concluding remarks in Section 7.

\subsection{Notation}

For a bounded Lipschitz domain $D$ in $\mathbb{R}^{d}, d \geq 1$, we denote by $L^{p}(D)$, $1 \leq p \leq \infty$, the Lebesgue space of $p$-integrable functions, endowed with the norm $\|\cdot\|_{L^{p}(D)}$. We set $L_{0}^{2}(D):=\left\{q \in L^{2}(D): \int_{D} q d \mathbf{x}=0\right\}$. The space of $p$-times continuously differentiable functions on $D$ is $C^{p}(\bar{D}), 0 \leq p \leq \infty$, equipped with the usual norm $\|\cdot\|_{C^{p}(\bar{D})}$. The standard Sobolev space of functions with integer or fractional regularity exponent $s>0$ is denoted by $H^{s}(D)$. We write $\|\cdot\|_{H^{s}(D)}$ and $|\cdot|_{H^{s}(D)}$ for its norm and seminorm, respectively, and set $H^{0}(D)=L^{2}(D)$. The trace space of $H^{1}(D)$ is denoted by $H^{\frac{1}{2}}(\partial D)$ and, as usual, we define $H_{0}^{1}(D)$ as the subspace of functions in $H^{1}(D)$ with zero trace on $\partial D$. The dual space of $H_{0}^{1}(D)$ is denoted by $H^{-1}(D)$. For a function space $X(D)$ we write $X(D)^{d}$ and $X(D)^{d \times d}$ to denote vector and tensor fields whose components belong to $X(D)$, respectively. Without further specification, these spaces are equipped with the usual product norms (which we simply denote by $\|\cdot\|_{X(D)}$ ). For vectors $\mathbf{v}, \mathbf{w} \in \mathbb{R}^{d}$, and matrices $\underline{\sigma}, \underline{\tau} \in \mathbb{R}^{d \times d}$, we use the standard notation $(\nabla \mathbf{v})_{i j}=\partial_{j} v_{i}$, $(\nabla \cdot \underline{\sigma})_{i}=\sum_{j=1}^{\bar{d}} \partial_{j} \sigma_{i j}$, and $\underline{\sigma}: \underline{\tau}=\sum_{i, j=1}^{d} \sigma_{i j} \tau_{i j}$. Furthermore, we denote by $\mathbf{v} \otimes \mathbf{w}$ the matrix whose $i j$-th component is $v_{i} w_{j}$, and use the identity $\mathbf{v} \cdot \underline{\sigma} \cdot \mathbf{w}=\sum_{i, j=1}^{d} v_{i} \sigma_{i j} w_{j}=\underline{\sigma}:(\mathbf{v} \otimes \mathbf{w})$. 


\section{The Stokes problem with piecewise analytic data}

\subsection{The Stokes equations}

Let $\Omega \subset \mathbb{R}^{2}$ be a polygonal and bounded domain. The Stokes problem is to find a velocity field $\mathbf{u}$ and a pressure $p$ such that

$$
\begin{aligned}
& -\Delta \mathbf{u}+\nabla p=\mathbf{f} \text { in } \Omega, \\
& \nabla \cdot \mathbf{u}=0 \text { in } \Omega, \\
& \mathbf{u}=\mathbf{g} \quad \text { on } \partial \Omega \text {. }
\end{aligned}
$$

Here, the right-hand side $\mathbf{f} \in H^{-1}(\Omega)^{2}$ is an exterior body force, and $\mathbf{g} \in$ $H^{\frac{1}{2}}(\partial \Omega)^{2}$ a prescribed Dirichlet datum satisfying the compatibility condition $\int_{\partial \Omega} \mathbf{g} \cdot \mathbf{n} d s=0$, with $\mathbf{n}$ denoting the outward unit normal vector to $\partial \Omega$.

The mixed variational form of the Stokes equations reads as follows: find $(\mathbf{u}, p) \in H^{1}(\Omega)^{2} \times L_{0}^{2}(\Omega)$, with $\mathbf{u}=\mathbf{g}$ on $\partial \Omega$, such that

$$
\left\{\begin{aligned}
\int_{\Omega} \nabla \mathbf{u}: \nabla \mathbf{v} d \mathbf{x}-\int_{\Omega} p \nabla \cdot \mathbf{v} d \mathbf{x} & =\langle\mathbf{f}, \mathbf{v}\rangle \\
\int_{\Omega} q \nabla \cdot \mathbf{u} d \mathbf{x} & =0
\end{aligned}\right.
$$

for all $(\mathbf{v}, q) \in H_{0}^{1}(\Omega)^{2} \times L_{0}^{2}(\Omega)$. Here, $\langle\cdot, \cdot\rangle$ denotes the standard duality pairing in $H^{-1}(\Omega)^{2} \times H_{0}^{1}(\Omega)^{2}$. It is well known that, due to the continuous inf-sup condition, the Stokes system has a unique solution $(\mathbf{u}, p)$ in $H^{1}(\Omega)^{2} \times L_{0}^{2}(\Omega)$; see, e.g., $[9,17]$ for details.

\subsection{Analytic regularity in polygonal domains}

For piecewise analytic data, the regularity of the exact solution $(\mathbf{u}, p)$ of the Stokes system was recently described by Guo and Schwab [23] in terms of the weighted Sobolev spaces that were originally introduced by Babuška and Guo for closely related elasticity and potential problems; see [20,21, 19,2-4, $22,30]$ and the references cited therein. To define these weighted spaces, let $\left\{A_{i}\right\}_{i=1}^{M}$ denote the vertices of the domain $\Omega$. To each vertex $A_{i}$ we assign a weight $\beta_{i} \geq 0$ and store these numbers in the $M$-tuple $\beta=\left(\beta_{1}, \ldots, \beta_{M}\right)$. We define $\beta \pm j:=\left(\beta_{1} \pm j, \ldots, \beta_{M} \pm j\right)$ and use the shorthand notation $C_{1}>\beta>C_{2}$ to mean $C_{1}>\beta_{i}>C_{2}$ for $i=1, \ldots, M$. For $r_{i}^{*}(\mathbf{x})=$ $\min \left\{1,\left|\mathbf{x}-A_{i}\right|\right\}$ we define the weight function $\Phi_{\underline{\beta}}(\mathbf{x}):=\prod_{i=1}^{M} r_{i}^{*}(\mathbf{x})^{\beta_{i}}$, and introduce the seminorms

$$
|u|_{H_{\underline{\beta}}^{k, l}(\Omega)}^{2}:=\sum_{|\alpha| \geq l}^{k}\left\|\Phi_{\underline{\beta}+|\alpha|-l} D^{\alpha} u\right\|_{L^{2}(\Omega)}^{2}, \quad k \geq l \geq 0 .
$$


We denote by $H_{\beta}^{k, l}(\Omega)$ the completion of $C^{\infty}(\bar{\Omega})$ with respect to the norm

$$
\begin{aligned}
& \|u\|_{H_{\underline{\beta}}^{k, l}(\Omega)}^{2}:=\|u\|_{H^{l-1}(\Omega)}^{2}+|u|_{H_{\underline{\beta}}^{k, l}(\Omega)}^{2}, \quad l \geq 1, \\
& \|u\|_{H_{\underline{\beta}}^{k, 0}(\Omega)}^{2}:=\sum_{|\alpha|=0}^{k}\left\|\Phi_{\underline{\beta}+|\alpha|} D^{\alpha} u\right\|_{L^{2}(\Omega)}^{2} .
\end{aligned}
$$

Definition 1 For an $M$-tuple $\underline{\beta}=\left(\beta_{1}, \ldots, \beta_{M}\right)$ and $l \geq 0$, the countably normed space $B_{\underline{\beta}}^{l}(\Omega)$ consists of all functions $u$ for which $u \in H_{\underline{\beta}}^{k, l}(\Omega)$ for $k \geq l$ and

$$
\left\|\Phi_{\underline{\beta}+k-l} D^{\alpha} u\right\|_{L^{2}(\Omega)} \leq C d^{(k-l)}(k-l) !, \quad|\alpha|=k \geq l,
$$

for some constants $C>0, d \geq 1$ independent of $k$.

We remark that $B_{\underline{\beta}}^{2}(\Omega) \not \subset H^{2}(\Omega)$ and $B_{\underline{\beta}}^{1}(\Omega) \not \subset H^{1}(\Omega)$. However, $B_{\underline{\beta}}^{2}(\Omega) \subset C^{0}(\bar{\Omega})$ and $B_{\underline{\beta}}^{1}\left(\Omega_{\text {int }}\right) \subset C^{0}\left(\bar{\Omega}_{\text {int }}\right)$, for all interior domains $\Omega_{\text {int }}$ with $\bar{\Omega}_{\text {int }} \subset \bar{\Omega} \backslash\left\{A_{i}\right\}_{i=1}^{M}$.

For a noninteger exponent $k$, the space $H_{\beta}^{k, l}(\Omega)$ is defined by interpolation. Finally, we define $H_{\underline{\beta}}^{k-\frac{1}{2}, l-\frac{1}{2}}(\partial \Omega)$ and $B_{\underline{\beta}}^{l-\frac{1}{2}}(\partial \Omega)$ as spaces of traces of functions in $H_{\underline{\beta}}^{k, l}(\Omega)$ and $B_{\underline{\beta}}^{l}(\Omega)$, respectively. The space $H_{\underline{\beta}}^{k-\frac{1}{2}, l-\frac{1}{2}}(\partial \Omega)$ is endowed with the norm

$$
\|g\|_{H_{\underline{\beta}}^{k-\frac{1}{2}, l-\frac{1}{2}}(\partial \Omega)}=\inf \left\{\|u\|_{H_{\underline{\beta}}^{k, l}(\Omega)}:\left.u\right|_{\partial \Omega}=g\right\} .
$$

The following regularity result will be the basis of our analysis; its proof can be found in [23].

Theorem 1 There exist a weight vector $0 \leq \underline{\beta}_{\min }<1$ depending on the opening angles of $\Omega$ at the vertices $\left\{A_{i}\right\}_{i=1}^{M}$ such that for weight vectors $\beta$ with $\underline{\beta}_{\min }<\underline{\beta}<1$ and piecewise analytic data

$$
\mathbf{f} \in B_{\underline{\beta}}^{0}(\Omega)^{2}, \quad \mathbf{g} \in B_{\underline{\beta}}^{\frac{3}{2}}(\partial \Omega)^{2},
$$

the solution $(\mathbf{u}, p)$ of the Stokes system satisfies

$$
\mathbf{u} \in B_{\underline{\beta}}^{2}(\Omega)^{2}, \quad p \in B_{\underline{\beta}}^{1}(\Omega) .
$$

We point out that, in particular, Theorem 1 implies that

$$
\mathbf{u} \in H_{\underline{\beta}}^{2,2}(\Omega)^{2}, \quad p \in H_{\underline{\beta}}^{1,1}(\Omega), \quad \nabla \mathbf{u} \in H_{\underline{\beta}}^{1,1}(\Omega)^{2 \times 2},
$$

and

$$
-\Delta \mathbf{u}+\nabla p=\mathbf{f} \quad \text { in } H_{\underline{\beta}}^{0,0}(\Omega)^{2} .
$$

Throughout the paper, the smoothness property in Theorem 1 is assumed to hold for a weight vector $\underline{\beta}$ with $\underline{\beta}_{\min }<\underline{\beta}<1$. 


\section{Meshes and trace operators}

In this section, we introduce the trace operators that are needed to define the interelemental terms in the DGFEM. Furthermore, we prove a series of technical results that allow us to properly treat the elements at the vertices of the computational domain. Similar results were used recently in $[35,34]$ to analyze $h p$-DGFEM for diffusion and elasticity problems.

\subsection{Meshes}

Throughout, we assume that the domain $\Omega$ can be subdivided into shaperegular affine meshes $\mathcal{T}_{h}=\{K\}$ consisting of parallelograms $K$. For each $K \in \mathcal{T}_{h}$, we denote by $\mathbf{n}_{K}$ the outward unit normal vector to the boundary $\partial K$, and by $h_{K}$ the elemental diameter. Furthermore, we assign to each element $K \in \mathcal{T}_{h}$ an approximation order $k_{K} \geq 1$. The local quantities $h_{K}$ and $k_{K}$ are stored in the vectors $\underline{h}=\left\{h_{K}\right\}_{K \in \mathcal{T}_{h}}$ and $\underline{k}=\left\{k_{K}\right\}_{K \in \mathcal{T}_{h}}$, respectively. We set $h=\max _{K \in \mathcal{T}_{h}} h_{K}$ and $|\underline{k}|=\max _{K \in \mathcal{T}_{h}} k_{K}$.

An interior edge of $\mathcal{T}_{h}$ is the (non-empty) one-dimensional interior of $\partial K^{+} \cap \partial K^{-}$, where $K^{+}$and $K^{-}$are two adjacent elements of $\mathcal{T}_{h}$. Similarly, a boundary edge of $\mathcal{T}_{h}$ is the (non-empty) one-dimensional interior of $\partial K \cap \partial \Omega$ which consists of entire edges of $\partial K$. We denote by $\mathcal{E}_{\mathcal{I}}$ the union of all interior edges of $\mathcal{T}_{h}$, by $\mathcal{E}_{\mathcal{D}}$ the union of all boundary edges, and set $\mathcal{E}=\mathcal{E}_{\mathcal{I}} \cup \mathcal{E}_{\mathcal{D}}$. Generally, we allow for irregular meshes, i.e., meshes with hanging nodes (see [30, Sect. 4.4.1]), but suppose that the intersection between neighboring elements is either a common vertex or a common edge of one of the two elements. We also assume the local mesh sizes and approximation degrees to be of bounded variation: that is, there is a constant $\kappa>0$ such that

$$
\kappa h_{K} \leq h_{K^{\prime}} \leq \kappa^{-1} h_{K}, \quad \kappa k_{K} \leq k_{K^{\prime}} \leq \kappa^{-1} k_{K},
$$

whenever $K$ and $K^{\prime}$ share a common edge.

\subsection{Averages and jumps}

Next, we define average and jump operators. To this end, let $K^{+}$and $K^{-}$be two adjacent elements of $\mathcal{T}_{h}$; let $\mathbf{x}$ be an arbitrary point of the interior edge $e=\partial K^{+} \cap \partial K^{-} \subset \mathcal{E}_{\mathcal{I}}$. Let $q$, v, and $\underline{\tau}$ be scalar-, vector-, and matrix-valued functions, respectively, that are smooth inside each element $K^{ \pm}$, and let us denote by $\left(q^{ \pm}, \mathbf{v}^{ \pm}, \underline{\tau}^{ \pm}\right)$the traces of $(q, \mathbf{v}, \underline{\tau})$ on $e$ taken from within the interior of $K^{ \pm}$. Then, we define the following averages at $\mathbf{x} \in e$

$$
\left\{\{q\}=\left(q^{+}+q^{-}\right) / 2, \quad\left\{\{\mathbf{v}\}=\left(\mathbf{v}^{+}+\mathbf{v}^{-}\right) / 2, \quad\left\{\{\underline{\tau}\}=\left(\underline{\tau}^{+}+\underline{\tau}^{-}\right) / 2 .\right.\right.\right.
$$


Similarly, the jumps at $\mathbf{x} \in e$ are given by

$$
\begin{array}{lll}
\llbracket q \rrbracket=q^{+} \mathbf{n}_{K^{+}}+q^{-} \mathbf{n}_{K^{-}}, & & \llbracket \mathbf{v} \rrbracket=\mathbf{v}^{+} \cdot \mathbf{n}_{K^{+}}+\mathbf{v}^{-} \cdot \mathbf{n}_{K^{-}}, \\
\underline{\llbracket \mathbf{v} \rrbracket}=\mathbf{v}^{+} \otimes \mathbf{n}_{K^{+}}+\mathbf{v}^{-} \otimes \mathbf{n}_{K^{-}}, & & \llbracket \tau \rrbracket=\underline{\tau}{ }^{+} \mathbf{n}_{K^{+}}+\underline{\tau}{ }^{-} \mathbf{n}_{K^{-}} .
\end{array}
$$

On boundary edges $e \subset \mathcal{E}_{\mathcal{D}}$, we set $\{\{q\}\}=q$, $\{\{\mathbf{v}\}\}=\mathbf{v},\{\{\underline{\tau}\}\}=\underline{\tau}$, as well as $\llbracket q \rrbracket=q \mathbf{n}, \llbracket \mathbf{v} \rrbracket=\mathbf{v} \cdot \mathbf{n}, \underline{\llbracket \mathbf{v} \rrbracket}=\mathbf{v} \otimes \mathbf{n}$, and $\llbracket \underline{\tau} \rrbracket=\underline{\tau} \mathbf{n}$.

\subsection{Elements near vertices}

To account for the singular behavior of solutions near the vertices $\left\{A_{i}\right\}_{i=1}^{M}$ of the domain, we define the sets

$$
\begin{aligned}
\mathcal{K}_{\text {vert }} & =\left\{K \in \mathcal{T}_{h}: \bar{K} \cap A_{i} \neq \emptyset \text { for some } 1 \leq i \leq M\right\}, \\
\mathcal{K}_{\text {int }} & =\left\{K \in \mathcal{T}_{h}: \bar{K} \cap A_{i}=\emptyset \text { for all } 1 \leq i \leq M\right\}
\end{aligned}
$$

Let $K \in \mathcal{K}_{\text {vert }}$. We always assume that the partitions $\mathcal{T}_{h}$ are fine enough so that exactly one vertex belongs to element $K$. We denote this vertex by $A_{K}$ and the corresponding weight exponent by $\beta_{K} \in[0,1)$. The spaces $H_{\beta_{K}}^{k, \ell}(K)$ are defined as in Section 2, but equipped with the weight function $\Phi_{\beta_{K}}(\mathbf{x})=r^{\beta_{K}}$, with $r$ denoting the distance to the corner $A_{K}$. We have the following auxiliary results.

Lemma 1 Let $K \in \mathcal{K}_{\text {vert. Then: }}$

1. We have $H_{\beta_{K}}^{0,0}(K) \subset L^{1}(K)$ and

$$
\|\varphi\|_{L^{1}(K)} \leq C h_{K}^{1-\beta_{K}}\|\varphi\|_{H_{\beta_{K}}^{0,0}(K)}, \quad \forall \varphi \in H_{\beta_{K}}^{0,0}(K) .
$$

2. Let $\varphi \in H_{\beta_{K}}^{0,0}(K)$ and $v \in L^{\infty}(K)$. Then the integral $\int_{K} \varphi v d \mathbf{x}$ is welldefined and $\left|\int_{K} \varphi v d \mathbf{x}\right| \leq C h_{K}^{1-\beta_{K}}\|v\|_{L^{\infty}(K)}\|\varphi\|_{H_{\beta_{K}}^{0,0}(K)}$.

3. Let $\varphi \in H_{\beta_{K}}^{1,1}(K)$. Then the trace $\left.\varphi\right|_{\partial K}$ belongs to $L^{1}(\partial K)$ and satisfies

$$
\|\varphi\|_{L^{1}(\partial K)} \leq C\left(\|\varphi\|_{L^{2}(K)}+h_{K}^{1-\beta_{K}}|\varphi|_{H_{\beta_{K}}^{1,1}(K)}\right) .
$$

All the constants $C>0$ are independent of $\underline{h}$ and of $\underline{k}$.

Proof. For $\varphi \in H_{\beta_{K}}^{0,0}(K)$, we have

$$
\int_{K}|\varphi| d \mathbf{x} \leq\left\|r^{-\beta_{K}}\right\|_{L^{2}(K)}\left\|r^{\beta_{K}} \varphi\right\|_{L^{2}(K)}=\left\|r^{-\beta_{K}}\right\|_{L^{2}(K)}\|\varphi\|_{H_{\beta_{K}}^{0,0}(K)} .
$$

Since $\left\|r^{-\beta_{K}}\right\|_{L^{2}(K)} \leq C h_{K}^{1-\beta_{K}}$, the first assertion follows. The second assertion follows then straightforwardly from Hölder's inequality. To prove the 
third assertion, let $\varphi \in H_{\beta_{K}}^{1,1}(K)$. From the standard trace theorem and a scaling argument, we have

$$
\|\varphi\|_{L^{1}(\partial K)} \leq C\left(h_{K}^{-1}\|\varphi\|_{L^{1}(K)}+\|\nabla \varphi\|_{L^{1}(K)}\right) .
$$

First, we note that $h_{K}^{-1}\|\varphi\|_{L^{1}(K)} \leq C\|\varphi\|_{L^{2}(K)}$. Next, since $\nabla \varphi \in H_{\beta_{K}}^{0,0}(K)^{2}$, we have $\|\nabla \varphi\|_{L^{1}(K)} \leq C h_{K}^{1-\beta_{K}}|\varphi|_{H_{\beta_{K}}^{1,1}(K)}$, which is a consequence of the first assertion and the definition of the seminorm $|\cdot|_{H_{\beta_{K}}^{1,1}(K)}$. This completes the proof.

Lemma 2 Let $K \in \mathcal{K}_{\text {vert }}, \underline{\tau} \in H_{\beta_{K}}^{1,1}(K)^{2 \times 2}$ and $\mathbf{v} \in C^{1}(\bar{K})^{2}$. Then the following integration by parts formula holds

$$
\int_{K} \nabla \cdot \tau \cdot \mathbf{v} d \mathbf{x}=-\int_{K} \underline{\tau}: \nabla \mathbf{v} d \mathbf{x}+\int_{\partial K} \underline{\tau}:\left(\mathbf{v} \otimes \mathbf{n}_{K}\right) d s,
$$

where the term on the left and the boundary term are understood as $L^{1} \times L^{\infty}$ pairings.

Proof. We start by noting that all the integrals above are well-defined due to Lemma 1 and the fact that $\nabla \cdot \underline{\tau} \in H_{\beta_{K}}^{0,0}(K)^{2}$. Furthermore, since $C^{\infty}(\bar{K})$ is dense in $H_{\beta_{K}}^{1,1}(K)$, there exists a sequence $\left\{\underline{\tau}_{n}\right\} \subset C^{\infty}(\bar{K})^{2 \times 2}$ with $\underline{\tau}_{n} \rightarrow \underline{\tau}$ in $H_{\beta_{K}}^{1,1}(K)^{2 \times 2}$. Clearly,

$$
\int_{K} \nabla \cdot \tau_{n} \cdot \mathbf{v} d \mathbf{x}=-\int_{K} \underline{\tau}_{n}: \nabla \mathbf{v} d \mathbf{x}+\int_{\partial K} \underline{\tau}_{n}:\left(\mathbf{v} \otimes \mathbf{n}_{K}\right) d s .
$$

The trace estimate from Lemma 1 yields

$$
\left|\int_{\partial K}\left(\underline{\tau}-\underline{\tau}_{n}\right):\left(\mathbf{v} \otimes \mathbf{n}_{K}\right) d s\right| \leq C\|\mathbf{v}\|_{L^{\infty}(\partial K)}\left\|\underline{\tau}-\underline{\tau}_{n}\right\|_{H_{\beta_{K}}^{1,1}(K)} .
$$

Furthermore, again with Lemma 1,

$$
\begin{aligned}
\left|\int_{K} \nabla \cdot\left(\underline{\tau}-\underline{\tau}_{n}\right) \cdot \mathbf{v} d \mathbf{x}\right| & \leq\|\mathbf{v}\|_{L^{\infty}(K)}\left\|\nabla \cdot\left(\underline{\tau}-\underline{\tau}_{n}\right)\right\|_{L^{1}(K)} \\
& \leq C h_{K}^{1-\beta_{K}}\|\mathbf{v}\|_{L^{\infty}(K)}\left\|\underline{\tau}-\underline{\tau}_{n}\right\|_{H_{\beta_{K}}^{1,1}(K)},
\end{aligned}
$$

and

$$
\begin{aligned}
\left|\int_{K}\left(\underline{\tau}-\underline{\tau}_{n}\right): \nabla \mathbf{v} d \mathbf{x}\right| & \leq\|\nabla \mathbf{v}\|_{L^{2}(K)}\left\|\underline{\tau}-\underline{\tau}_{n}\right\|_{L^{2}(K)} \\
& \leq\|\nabla \mathbf{v}\|_{L^{2}(K)}\left\|\underline{\tau}-\underline{\tau}_{n}\right\|_{H_{\beta_{K}}^{1,1}(K)} .
\end{aligned}
$$

Passing to the limit finishes the proof. 
Lemma 3 Let the exact solution $(\mathbf{u}, p)$ of the Stokes system satisfy (4). For an interior edge $e \subset \mathcal{E}_{\mathcal{I}}$, we have that $\llbracket \nabla \mathbf{u}-p \underline{I} \rrbracket=\mathbf{0}$ on $e$.

Proof. We first note that $\nabla \mathbf{u}-p \underline{I}$ belongs to $C^{0}\left(\bar{\Omega}_{\text {int }}\right)$ for all interior domains $\Omega_{\text {int }}$ with $\bar{\Omega}_{\text {int }} \subset \bar{\Omega} \backslash\left\{A_{i}\right\}_{i=1}^{M}$. Hence, if $\bar{e} \cap\left\{A_{i}\right\}_{i=1}^{M}=\emptyset$, we immediately have that $\llbracket \nabla \mathbf{u}-p \underline{I \rrbracket}=\mathbf{0}$ on $e$. Let us next consider the case where $\bar{e} \cap\left\{A_{i}\right\}_{i=1}^{M}=A_{j}$ for a vertex $A_{j}$. We may assume that the edge is parameterized by $\bar{e}=\varphi(t)$, $t \in[0,1]$, with $\varphi(0)=A_{j}$. Then,

$$
\int_{\varepsilon}^{1}|\llbracket \nabla \mathbf{u}-p \underline{I} \rrbracket|\left|\varphi^{\prime}(t)\right| d t=0,
$$

for all $\varepsilon>0$. Thanks to (5), we have $\nabla \mathbf{u}-p \underline{I} \in H_{\beta}^{1,1}(\Omega)^{2 \times 2}$. Thus, $\mathbb{I} \nabla \mathbf{u}-$ $p \underline{I]} \in L^{1}(e)^{2}$, according to Lemma 1 . We conclude with the dominated convergence theorem that

$$
\int_{0}^{1}|\llbracket \nabla \mathbf{u}-p \underline{I} \rrbracket|\left|\varphi^{\prime}(t)\right| d t=0,
$$

and thus $\llbracket \nabla \mathbf{u}-p \underline{I} \rrbracket=\mathbf{0}$ on $e$.

\section{Discontinuous Galerkin discretization}

In this section, we introduce discontinuous Galerkin methods for the Stokes problem and review their well-posedness, using the recent results in [29].

\subsection{Mixed DGFEM}

Given a mesh $\mathcal{T}_{h}$ and a degree vector $\underline{k}=\left\{k_{K}\right\}, k_{K} \geq 1$, we approximate the Stokes problem by finite element functions $\left(\mathbf{u}_{h}, p_{h}\right) \in \mathbf{V}_{h} \times Q_{h}$ where

$$
\begin{aligned}
& \mathbf{V}_{h}=\left\{\mathbf{v} \in L^{2}(\Omega)^{2}:\left.\mathbf{v}\right|_{K} \in \mathcal{Q}^{k_{K}}(K)^{2}, K \in \mathcal{T}_{h}\right\}, \\
& Q_{h}=\left\{q \in L_{0}^{2}(\Omega):\left.q\right|_{K} \in \mathcal{Q}^{k_{K}-1}(K), K \in \mathcal{T}_{h}\right\} .
\end{aligned}
$$

Here, $\mathcal{Q}^{k}(K)$ denotes the space of polynomials of degree at most $k \geq 0$ in each variable on $K$. For further reference, we also define the space

$$
\widetilde{Q}_{h}=\left\{q \in L^{2}(\Omega):\left.q\right|_{K} \in \mathcal{Q}^{k_{K}-1}(K), K \in \mathcal{T}_{h}\right\} .
$$

We consider the mixed method: find $\left(\mathbf{u}_{h}, p_{h}\right) \in \mathbf{V}_{h} \times Q_{h}$ such that

$$
\left\{\begin{aligned}
A_{h}\left(\mathbf{u}_{h}, \mathbf{v}\right)+B_{h}\left(\mathbf{v}, p_{h}\right) & =F_{h}(\mathbf{v}) \\
-B_{h}\left(\mathbf{u}_{h}, q\right) & =G_{h}(q)
\end{aligned}\right.
$$


for all $(\mathbf{v}, q) \in \mathbf{V}_{h} \times Q_{h}$. The forms $A_{h}$ and $B_{h}$ are discontinuous Galerkin forms that discretize the Laplacian and the incompressibility constraint, respectively, with corresponding right-hand sides $F_{h}$ and $G_{h}$. These forms are given by

$$
\begin{aligned}
& A_{h}(\mathbf{u}, \mathbf{v})=\int_{\Omega} \nabla_{h} \mathbf{u}: \nabla_{h} \mathbf{v} d \mathbf{x}-\int_{\mathcal{E}}\left(\left\{\left\{\nabla_{h} \mathbf{v}\right\}\right\}: \underline{\llbracket \mathbf{u} \rrbracket}+\left\{\left\{\nabla_{h} \mathbf{u}\right\}\right\}: \underline{\llbracket \mathbf{v} \rrbracket}\right) d s \\
& +\int_{\mathcal{E}} \mathrm{c} \underline{\llbracket \mathbf{u} \rrbracket}: \underline{\llbracket \mathbf{v} \rrbracket} d s, \\
& B_{h}(\mathbf{v}, q)=-\int_{\Omega} q \nabla_{h} \cdot \mathbf{v} d \mathbf{x}+\int_{\mathcal{E}}\{\{q\} \llbracket \mathbf{v} \rrbracket d s, \\
& F_{h}(\mathbf{v})=\int_{\Omega} \mathbf{f} \cdot \mathbf{v} d \mathbf{x}-\int_{\mathcal{E}_{\mathcal{D}}}(\mathbf{g} \otimes \mathbf{n}): \nabla_{h} \mathbf{v} d s+\int_{\mathcal{E}_{\mathcal{D}}} \mathrm{c} \mathbf{g} \cdot \mathbf{v} d s, \\
& G_{h}(q)=-\int_{\mathcal{E}_{\mathcal{D}}} q \mathbf{g} \cdot \mathbf{n} d s .
\end{aligned}
$$

Here, $\nabla_{h}$ and $\nabla_{h}$. denote the discrete gradient and divergence operator, taken elementwise. The function $\mathrm{c} \in L^{\infty}(\mathcal{E})$ is the so-called discontinuity stabilization function that is chosen as follows. Define the functions $\mathrm{h} \in L^{\infty}(\mathcal{E})$ and $\mathrm{k} \in L^{\infty}(\mathcal{E})$ by

$$
\begin{aligned}
\mathrm{h}(\mathbf{x}) & := \begin{cases}\min \left\{h_{K}, h_{K^{\prime}}\right\}, & \mathbf{x} \in e=\partial K \cap \partial K^{\prime} \subset \mathcal{E}_{\mathcal{I}}, \\
h_{K}, & \mathbf{x} \in e=\partial K \cap \partial \Omega \subset \mathcal{E}_{\mathcal{D}},\end{cases} \\
\mathrm{k}(\mathbf{x}) & := \begin{cases}\max \left\{k_{K}, k_{K^{\prime}}\right\}, & \mathbf{x} \in e=\partial K \cap \partial K^{\prime} \subset \mathcal{E}_{\mathcal{I}}, \\
k_{K}, & \mathbf{x} \in e=\partial K \cap \partial \Omega \subset \mathcal{E}_{\mathcal{D}} .\end{cases}
\end{aligned}
$$

Then we set

$$
c=\gamma \mathrm{h}^{-1} \mathrm{k}^{2},
$$

with a parameter $\gamma>0$ that is independent of $\mathrm{h}$ and $\mathrm{k}$.

Remark 1 It can be seen from (5) and the trace properties in Lemma 1 that the forms $A_{h}$ and $B_{h}$ are well-defined when inserting the exact solution (u, $p$ ) satisfying (4). Similarly, $F_{h}$ and $G_{h}$ are well-defined due to (3) and Lemma 1.

Remark 2 The form $A_{h}$ corresponds to the so-called symmetric interior penalty discretization of the Laplace operator; see [1] and [29] where the presentation and analysis of several different DG methods were unified for diffusion problems and the Stokes system, respectively. We emphasize that all the results presented in this paper hold true verbatim for all the mixed discontinuous Galerkin methods investigated in [29]. 


\subsection{Well-posedness and basic error estimates}

Well-posedness of the discrete system (8) was established in [29], together with basic error estimates for the approximate solutions. To discuss this, we introduce the space

$$
\mathbf{V}(h):=\mathbf{V}_{h}+H^{1}(\Omega)^{2},
$$

endowed with the broken norm

$$
\|\mathbf{v}\|_{h}^{2}=\left\|\nabla_{h} \mathbf{v}\right\|_{L^{2}(\Omega)}^{2}+\int_{\mathcal{E}} \mathrm{h}^{-1} \mathrm{k}^{2}|\underline{\underline{\llbracket \mathbf{v}} \|}|^{2} d s, \quad \mathbf{v} \in \mathbf{V}(h) .
$$

We first note that the forms $A_{h}$ and $B_{h}$ are continuous on $\mathbf{V}_{h}$ and $Q_{h}$, that is

$$
\begin{aligned}
\left|A_{h}(\mathbf{v}, \mathbf{w})\right| & \leq C\|\mathbf{v}\|_{h}\|\mathbf{w}\|_{h}, & & \mathbf{v}, \mathbf{w} \in \mathbf{V}_{h} \\
\left|B_{h}(\mathbf{v}, q)\right| & \leq C\|\mathbf{v}\|_{h}\|q\|_{L^{2}(\Omega)}, & & \mathbf{v} \in \mathbf{V}_{h}, q \in Q_{h},
\end{aligned}
$$

with continuity constants $C>0$ independent of $\underline{h}$ and $\underline{k}$. Furthermore, there exists a parameter $\gamma_{\min }>0$ independent of $\underline{h}$ and $\underline{k}$ such that for any $\gamma \geq \gamma_{\min }$ there exists a coercivity constant $C>0$ independent of $\underline{h}$ and $\underline{k}$ with

$$
A_{h}(\mathbf{v}, \mathbf{v}) \geq C\|\mathbf{v}\|_{h}^{2}, \quad \mathbf{v} \in \mathbf{V}_{h} .
$$

Throughout, we assume that $\gamma \geq \gamma_{\min }$. Finally, for $k_{K} \geq 2$, the following discrete inf-sup condition for the finite element spaces $\mathbf{V}_{h}$ and $Q_{h}$ holds true:

$$
\inf _{0 \neq q \in Q_{h}} \sup _{\mathbf{0} \neq \mathbf{v} \in \mathbf{V}_{h}} \frac{B_{h}(\mathbf{v}, q)}{\|\mathbf{v}\|_{h}\|q\|_{L^{2}(\Omega)}} \geq C|\underline{k}|^{-1}>0,
$$

with a constant $C>0$ that is independent of $\underline{h}$ and $\underline{k}$.

The above properties of the forms $A_{h}$ and $B_{h}$, combined with well-known continuity properties for $F_{h}$ and $G_{h}$, show the well-posedness of the problem (8). The following abstract error bounds were obtained in [29, Sect. 3 and 4]: let $(\mathbf{u}, p)$ be the exact solution of the Stokes system and $\left(\mathbf{u}_{h}, p_{h}\right)$ the discontinuous Galerkin approximation (8). Then we have

$$
\begin{aligned}
\left\|\mathbf{u}-\mathbf{u}_{h}\right\|_{h} \leq & C|\underline{k}|\left[\inf _{\mathbf{w} \in \mathbf{V}_{h}}\|\mathbf{u}-\mathbf{w}\|_{h}+\inf _{q \in Q_{h}}\|p-q\|_{L^{2}(\Omega)}\right. \\
& \left.+\sup _{\mathbf{v} \in \mathbf{V}_{h}} \frac{\left|R_{h}(\mathbf{u}, p ; \mathbf{v})\right|}{\|\mathbf{v}\|_{h}}\right],
\end{aligned}
$$

as well as

$$
\begin{aligned}
\left\|p-p_{h}\right\|_{L^{2}(\Omega)} \leq & C|\underline{k}|^{2}\left[\inf _{q \in Q_{h}}\|p-q\|_{L^{2}(\Omega)}+\inf _{\mathbf{w} \in \mathbf{V}_{h}}\|\mathbf{u}-\mathbf{w}\|_{h}\right. \\
& \left.+\sup _{\mathbf{v} \in \mathbf{V}_{h}} \frac{\left|R_{h}(\mathbf{u}, p ; \mathbf{v})\right|}{\|\mathbf{v}\|_{h}}\right]
\end{aligned}
$$


where the constants $C>0$ are independent of $\underline{h}$ and $\underline{k}$. In the above estimates (10) and (11), the term $R_{h}(\mathbf{u}, p ; \mathbf{v})$ is a residual term that stems from the nonconformity of the method and is defined and investigated next.

To define the term $R_{h}(\mathbf{u}, p ; \mathbf{v})$, we introduce the auxiliary space

$$
\underline{\Sigma}_{h}:=\left\{\underline{\tau} \in L^{2}(\Omega)^{2 \times 2}: \underline{\tau} \in \mathcal{Q}^{k_{K}}(K)^{2 \times 2}, K \in \mathcal{T}_{h}\right\} .
$$

Moreover, we introduce the lifting operators $\underline{\mathcal{L}}: \mathbf{V}(h) \rightarrow \underline{\Sigma}_{h}$ and $\mathcal{M}$ : $\mathbf{V}(h) \rightarrow Q_{h}$ given by

$$
\begin{array}{rlrl}
\int_{\Omega} \underline{\mathcal{L}}(\mathbf{v}): \underline{\tau} d \mathbf{x}=\int_{\mathcal{E}} \underline{\llbracket \mathbf{v} \rrbracket}:\{\{\underline{\tau}\} d s, & & \forall \underline{\tau} \in \underline{\Sigma}_{h}, \\
\int_{\Omega} \mathcal{M}(\mathbf{v}) q d \mathbf{x}=\int_{\mathcal{E}} \llbracket \mathbf{v} \rrbracket\{\{q\}] d s, & \forall q \in Q_{h} .
\end{array}
$$

The residual can be expressed as follows; see [29] for details.

Lemma 4 Let $\mathbf{f} \in B_{\underline{\beta}}^{0}(\Omega)^{2}$. For test functions $\mathbf{v} \in \mathbf{V}_{h}$, we have

$$
\begin{aligned}
R_{h}(\mathbf{u}, p ; \mathbf{v})= & \int_{\Omega}[\nabla \mathbf{u}-p \underline{I}]: \nabla_{h} \mathbf{v} d \mathbf{x}-\int_{\Omega} \nabla \mathbf{u}: \underline{\mathcal{L}}(\mathbf{v}) d \mathbf{x} \\
& +\int_{\Omega} p \mathcal{M}(\mathbf{v}) d \mathbf{x}-\int_{\Omega} \mathbf{f} \cdot \mathbf{v} d \mathbf{x} .
\end{aligned}
$$

Remark 3 We point out that the regularity assumption (4) is not needed to obtain the abstract error estimates (10) and (11) and the expression for the residual in Lemma 4. The reason for this is that $A_{h}$ and $B_{h}$ can be extended in a non-consistent way to continuous forms on $\mathbf{V}(h) \times \mathbf{V}(h)$ and $\mathbf{V}(h) \times L^{2}(\Omega)$, respectively, with $\mathbf{V}(h)$ defined in (9); see [29] for details. The only assumption that is needed in Lemma 4 is that $\mathbf{f} \in B_{\beta}^{0}(\Omega)^{2}$ so as to make the integral $\int_{\Omega} \mathbf{f} \cdot \mathbf{v} d \mathbf{x}$ well-defined for a test function $\mathbf{v} \in \mathbf{V}_{h}$. We will invoke the regularity assumption (4) in the next section in order to show that $R_{h}$ is convergent.

Remark 4 For DG discretizations on triangular meshes, the explicit dependence of the discrete inf-sup condition on the approximation orders $\underline{k}$ seems not be known and remains to be investigated. A similar remark applies to non-affinely mapped quadrilaterals. This is the reason why we only consider meshes consisting of parallelograms, resulting in some restrictions on the types of polygons that can be subdivided accordingly.

\section{Error analysis}

In this section, we present an error analysis valid for piecewise analytic solutions. Special care is required for the elements $K \in \mathcal{K}_{\text {vert }}$ near the vertices. 


\subsection{The residual}

For piecewise smooth solutions, the residual expression in Lemma 4 has been shown to be optimally convergent in [29]. For solutions satisfying the regularity assumption (4) a more careful investigation is needed.

Lemma 5 Assume (3) and (4). Let $\underline{P}: L^{2}(\Omega)^{2 \times 2} \rightarrow \underline{\Sigma}_{h}$ and $P: L_{0}^{2}(\Omega) \rightarrow$ $Q_{h}$ denote the $L^{2}$-projections onto $\underline{\Sigma}_{h}$ and $Q_{h}$, respectively. Then we have

$$
R_{h}(\mathbf{u}, p ; \mathbf{v})=\int_{\mathcal{E}}\left\{\{\nabla \mathbf{u}-\underline{P}(\nabla \mathbf{u})\}: \underline{\llbracket \mathbf{v} \rrbracket} d s-\int_{\mathcal{E}}\{\{p-P(p)\}\} \llbracket \mathbf{v} \rrbracket d s\right.
$$

for all $\mathbf{v} \in \mathbf{V}_{h}$.

Proof. We first note that, by definition of the lifting operators,

$$
\left.\int_{\Omega} \nabla \mathbf{u}: \underline{\mathcal{L}}(\mathbf{v}) d \mathbf{x}=\int_{\Omega} \underline{P}(\nabla \mathbf{u}): \underline{\mathcal{L}}(\mathbf{v}) d \mathbf{x}=\int_{\mathcal{E}}\{\underline{P}(\nabla \mathbf{u})\}\right]: \underline{\llbracket \mathbf{v} \rrbracket} d s
$$

and

$$
\left.\int_{\Omega} p \mathcal{M}(\mathbf{v}) d \mathbf{x}=\int_{\Omega} P(p) \mathcal{M}(\mathbf{v}) d \mathbf{x}=\int_{\mathcal{E}}\{P(p)\}\right\} \llbracket \mathbf{v} \rrbracket d s .
$$

Furthermore, integrating by parts the expression in Lemma 4 over each element $K \in \mathcal{T}_{h}$ gives

$$
\begin{aligned}
R_{h}(\mathbf{u}, p ; \mathbf{v})= & \int_{\Omega}[-\Delta \mathbf{u}+\nabla p-\mathbf{f}] \cdot \mathbf{v} d \mathbf{x} \\
& +\sum_{K \in \mathcal{T}_{h}} \int_{\partial K}(\nabla \mathbf{u}-p \underline{I}):\left(\mathbf{v} \otimes \mathbf{n}_{K}\right) d s \\
& \left.-\int_{\mathcal{E}}\{\underline{P}(\nabla \mathbf{u})\}\right\}: \underline{\llbracket \mathbf{v} \rrbracket} d s+\int_{\mathcal{E}}\{\{P(p)\}\} \llbracket \mathbf{v} \rrbracket d s .
\end{aligned}
$$

Note that all the integrals are well-defined in view of Lemma 1, Lemma 2, (5) and (6). Elementary manipulations then show that

$$
\begin{aligned}
\sum_{K \in \mathcal{T}_{h}} \int_{\partial K}(\nabla \mathbf{u}-p \underline{I}):\left(\mathbf{v} \otimes \mathbf{n}_{K}\right) d s= & \left.\int_{\mathcal{E}_{\mathcal{I}}} \llbracket \nabla \mathbf{u}-p \underline{I} \rrbracket \cdot\{\mathbf{v}\}\right\} d s \\
& \left.+\int_{\mathcal{E}}\{\nabla \mathbf{u}-p \underline{I}\}\right\}: \underline{\llbracket \mathbf{v} \rrbracket} d s .
\end{aligned}
$$

Application of Lemma 3 yields

$$
\left.\sum_{K \in \mathcal{T}_{h}} \int_{\partial K}(\nabla \mathbf{u}-p \underline{I}):\left(\mathbf{v} \otimes \mathbf{n}_{K}\right) d s=\int_{\mathcal{E}}\{\nabla \nabla \mathbf{u}\}\right\}: \underline{\llbracket \mathbf{v} \rrbracket} d s-\int_{\mathcal{E}}\{\{p\} \llbracket \llbracket \mathbf{v} \rrbracket d s .
$$

Combining the above results and observing that $-\Delta \mathbf{u}+\nabla p=\mathbf{f}$ in $H_{\underline{\beta}}^{0,0}(\Omega)^{2}$, from (6), yields the assertion. 
We have the following estimate of $R_{h}$.

Lemma 6 Assume (3) and (4). For $\mathbf{v} \in \mathbf{V}_{h}$, we have

$$
\begin{aligned}
\left|R_{h}(\mathbf{u}, p ; \mathbf{v})\right| \leq & C\|\mathbf{v}\|_{h}\left[\|\mathbf{u}-\mathbf{w}\|_{h}+\|p-q\|_{L^{2}(\Omega)}\right] \\
& +\mid \int_{\mathcal{E}}\left\{\{\nabla \mathbf{u}-\nabla \mathbf{w}\}: \underline{\llbracket \mathbf{v} \rrbracket} d s-\int_{\mathcal{E}}\{\{p-q\}\} \llbracket \mathbf{v} \rrbracket d s \mid\right.
\end{aligned}
$$

for any $(\mathbf{w}, q) \in \mathbf{V}_{h} \times Q_{h}$.

Proof. Let $(\mathbf{w}, q) \in \mathbf{V}_{h} \times Q_{h}$ be arbitrary. From the result in Lemma 5 and since the $L^{2}$-projections reproduce polynomials in $\underline{\Sigma}_{h}$ and $Q_{h}$, respectively, we obtain

$$
\begin{aligned}
R_{h}(\mathbf{u}, p ; \mathbf{v})= & \int_{\mathcal{E}}\left\{\left\{\nabla \mathbf{u}-\nabla_{h} \mathbf{w}-\underline{P}\left(\nabla \mathbf{u}-\nabla_{h} \mathbf{w}\right)\right\}\right]: \underline{\llbracket \mathbf{v} \rrbracket} d s \\
& -\int_{\mathcal{E}}\{\{p-q-P(p-q)\}\} \llbracket \mathbf{v} \rrbracket d s .
\end{aligned}
$$

The term $T$ with the $L^{2}$-projections can be bounded by

$$
\begin{aligned}
|T|= & \left|\int_{\mathcal{E}}\left\{\underline{P}\left(\nabla \mathbf{u}-\nabla_{h} \mathbf{w}\right)\right\}: \underline{\llbracket \mathbf{v} \rrbracket} d s-\int_{\mathcal{E}}\{\{P(p-q)\}\} \llbracket \mathbf{v} \rrbracket d s\right| \\
\leq & C\|\mathbf{v}\|_{h} \sum_{K \in \mathcal{T}_{h}}\left[\frac{h_{K}}{k_{K}^{2}}\left\|\underline{P}\left(\nabla \mathbf{u}-\nabla_{h} \mathbf{w}\right)\right\|_{L^{2}(\partial K)}^{2}\right. \\
& \left.+\frac{h_{K}}{k_{K}^{2}}\|P(p-q)\|_{L^{2}(\partial K)}^{2}\right]^{1 / 2} \\
\leq & C\|\mathbf{v}\|_{h}\left[\left\|\underline{P}\left(\nabla \mathbf{u}-\nabla_{h} \mathbf{w}\right)\right\|_{L^{2}(\Omega)}+\|P(p-q)\|_{L^{2}(\Omega)}\right] \\
\leq & C\|\mathbf{v}\|_{h}\left[\|\mathbf{u}-\mathbf{w}\|_{h}+\|p-q\|_{L^{2}(\Omega)}\right] .
\end{aligned}
$$

Here, we used the Cauchy-Schwarz inequality, the definition of $h$ and $k$, the fact that $|\llbracket \mathbf{v} \rrbracket|^{2} \leq|\underline{\llbracket \mathbf{v} \rrbracket}|^{2}$, the discrete trace inequality

$$
\|\varphi\|_{L^{2}(\partial K)}^{2} \leq C k_{K}^{2} h_{K}^{-1}\|\varphi\|_{L^{2}(K)}^{2},
$$

valid for polynomials $\varphi \in \mathcal{Q}^{k_{K}}(K)$, and the stability of the $L^{2}$-projections. The triangle inequality completes the proof.

\subsection{Error estimates}

In this section, we combine the bounds (10) and (11) with the ones in Lemma 6 to obtain the following result. 
Theorem 2 Assume (3), let the exact solution (u, $p$ ) of the Stokes system satisfy (4), and let $\left(\mathbf{u}_{h}, p_{h}\right)$ be the discontinuous Galerkin approximation (8) with $k_{K} \geq 2$, for all $K \in \mathcal{T}_{h}$. Then, for any $(\mathbf{w}, \widetilde{q}) \in \mathbf{V}_{h} \times \widetilde{Q}_{h}$, we have the error bound

$$
\left\|\mathbf{u}-\mathbf{u}_{h}\right\|_{h}+\left\|p-p_{h}\right\|_{L^{2}(\Omega)} \leq C|\underline{k}|^{3}\left[E_{1}+E_{2}+E_{3}\right],
$$

where

$$
\begin{aligned}
& E_{1}^{2}=\sum_{K \in \mathcal{T}_{h}}\left[|\mathbf{u}-\mathbf{w}|_{H^{1}(K)}^{2}+h_{K}^{-2}\|\mathbf{u}-\mathbf{w}\|_{L^{2}(K)}^{2}+\|p-\widetilde{q}\|_{L^{2}(K)}^{2}\right], \\
& E_{2}^{2}=\sum_{K \in \mathcal{K}_{\text {int }}} h_{K}^{2}\left[|\mathbf{u}-\mathbf{w}|_{H^{2}(K)}^{2}+|p-\widetilde{q}|_{H^{1}(K)}^{2}\right], \\
& E_{3}^{2}=\sum_{K \in \mathcal{K}_{\text {vert }}} h_{K}^{2\left(1-\beta_{K}\right)}\left[|\mathbf{u}-\mathbf{w}|_{H_{\beta_{K}}^{2,2}(K)}^{2}+|p-\widetilde{q}|_{H_{\beta_{K}}^{1,1}(K)}^{2}\right] .
\end{aligned}
$$

The constant $C>0$ is independent of $\underline{h}$ and $\underline{k}$.

Proof. Let $\mathbf{w} \in \mathbf{V}_{h}, \widetilde{q} \in \widetilde{Q}_{h}$ be arbitrary. Set $q:=\widetilde{q}-\frac{1}{|\Omega|} \int_{\Omega} \widetilde{q} d \mathbf{x} \in Q_{h}$. Then, the bounds from (10), (11) and Lemma 6 yield

$$
\begin{aligned}
& \left\|\mathbf{u}-\mathbf{u}_{h}\right\|_{h}+\left\|p-p_{h}\right\|_{L^{2}(\Omega)} \\
& \leq C|\underline{k}|^{2}\left[\|\mathbf{u}-\mathbf{w}\|_{h}+\|p-q\|_{L^{2}(\Omega)}+\sup _{\mathbf{v} \in \mathbf{V}_{h}} \frac{\left|E_{h}(\mathbf{u}-\mathbf{w}, p-q ; \mathbf{v})\right|}{\|\mathbf{v}\|_{h}}\right],
\end{aligned}
$$

with $E_{h}$ given by

$$
\left.E_{h}(\mathbf{u}-\mathbf{w}, p-q ; \mathbf{v})=\int_{\mathcal{E}}\left\{\left\{\nabla \mathbf{u}-\nabla_{h} \mathbf{w}\right\}\right\}: \underline{\llbracket \mathbf{v} \rrbracket} d s-\int_{\mathcal{E}}\{p-q\}\right\} \llbracket \mathbf{v} \rrbracket d s .
$$

In the following, we estimate the right-hand side of (12) in terms of the error contributions $\left\{E_{i}\right\}_{i=1}^{3}$.

First, using the shape-regularity of the mesh, property (7), and the trace inequality

$$
\|\varphi\|_{L^{2}(\partial K)}^{2} \leq C\left[h_{K}^{-1}\|\varphi\|_{L^{2}(K)}^{2}+h_{K}|\varphi|_{H^{1}(K)}^{2}\right], \quad \forall \varphi \in H^{1}(K),
$$

valid with a constant $C>0$ independent of $\underline{h}$ and $\underline{k}$, yields

$$
\begin{aligned}
\|\mathbf{u}-\mathbf{w}\|_{h}^{2} & =\sum_{K \in \mathcal{T}_{h}}|\mathbf{u}-\mathbf{w}|_{H^{1}(K)}^{2}+\int_{\mathcal{E}} \mathrm{h}^{-1} \mathrm{k}^{2}|\underline{\llbracket \mathbf{u}-\mathbf{w} \|}|^{2} d s \\
& \leq \sum_{K \in \mathcal{T}_{h}}|\mathbf{u}-\mathbf{w}|_{H^{1}(K)}^{2}+C \sum_{K \in \mathcal{T}_{h}} h_{K}^{-1} k_{K}^{2}\|\mathbf{u}-\mathbf{w}\|_{L^{2}(\partial K)}^{2} \\
& \leq C|\underline{k}|^{2} \sum_{K \in \mathcal{T}_{h}}\left[h_{K}^{-2}\|\mathbf{u}-\mathbf{w}\|_{L^{2}(K)}^{2}+|\mathbf{u}-\mathbf{w}|_{H^{1}(K)}^{2}\right] \\
& \leq C|\underline{k}|^{2} E_{1}^{2} .
\end{aligned}
$$


Next, since $\int_{\Omega} p d \mathbf{x}=\int_{\Omega} q d \mathbf{x}=0$, we have

$$
\begin{aligned}
\|p-q\|_{L^{2}(\Omega)} & =\left\|p-\widetilde{q}-|\Omega|^{-1} \int_{\Omega}(p-\widetilde{q}) d \mathbf{x}\right\|_{L^{2}(\Omega)} \\
& \leq\|p-\widetilde{q}\|_{L^{2}(\Omega)}+|\Omega|^{-1 / 2} \int_{\Omega}|p-\widetilde{q}| d \mathbf{x} \\
& \leq 2\|p-\widetilde{q}\|_{L^{2}(\Omega)} \\
& \leq 2 E_{1} .
\end{aligned}
$$

Moreover,

$$
\begin{aligned}
& \left|E_{h}(\mathbf{u}-\mathbf{w}, p-q ; \mathbf{v})\right| \\
& \quad \leq \sum_{e \subset \mathcal{E}} \int_{e}\left[\left|\left\{\left\{\nabla \mathbf{u}-\nabla_{h} \mathbf{w}\right\}\right\}: \underline{\llbracket \mathbf{v} \rrbracket}\right|+|\{\{p-q\}\} \llbracket \mathbf{v} \rrbracket|\right] d s \\
& \quad \leq \sum_{e \subset \mathcal{E}} \int_{e}\left[\left|\left\{\left\{\nabla \mathbf{u}-\nabla_{h} \mathbf{w}\right\}\right\}\right|+|\{\{p-q\} \mid]| \underline{\underline{\llbracket \mathbf{v} \rrbracket} \mid} d s\right. \\
& \quad \leq \sum_{e \subset \mathcal{E}}\|\underline{\llbracket \mathbf{v} \rrbracket}\|_{L^{\infty}(e)} \int_{e}\left[\mid\left\{\mid \nabla \mathbf{u}-\nabla_{h} \mathbf{w}\right\}\right\}|+|\{\{p-q\} \mid] d s .
\end{aligned}
$$

Note that $\underline{\llbracket \mathbf{v} \rrbracket}$ is a polynomial on each edge $e \subset \mathcal{E}$. Applying a standard inverse inequality for polynomials (see, e.g., [27]) and using property (7) yields

$$
\begin{aligned}
\|\underline{\llbracket \mathbf{v} \rrbracket}\|_{L^{\infty}(e)} & =\left\|\left.\underline{\| \mathbf{\llbracket} \rrbracket}\right|^{2}\right\|_{L^{\infty}(e)}^{1 / 2} \\
& \leq C \frac{\left.\mathrm{k}\right|_{e}}{\sqrt{\left.\mathrm{h}\right|_{e}}}\left\|\left.\underline{\| \mathbf{v} \rrbracket}\right|^{2}\right\|_{L^{1}(e)}^{1 / 2} \leq C \frac{\left.\mathrm{k}\right|_{e}}{\sqrt{\left.\mathrm{h}\right|_{e}}}\|\underline{\llbracket \mathbf{v} \rrbracket}\|_{L^{2}(e)} .
\end{aligned}
$$

Therefore, using the shape-regularity of the mesh it follows that

$$
\begin{aligned}
\mid & E_{h}(\mathbf{u}-\mathbf{w}, p-q ; \mathbf{v}) \mid \\
\leq & C \sum_{e \subset \mathcal{E}}\left\|\frac{\mathrm{k}}{\sqrt{\mathrm{h}}} \underline{\llbracket \mathbf{v} \rrbracket}\right\|_{L^{2}(e)} \int_{e}\left[\left|\left\{\left\{\nabla \mathbf{u}-\nabla_{h} \mathbf{w}\right\}\right\}\right|+|\{\{p-q\}\}|\right] d s \\
\leq & C\left[\int_{\mathcal{E}} \mathrm{h}^{-1} \mathrm{k}^{2}|\underline{\llbracket \mathbf{v} \rrbracket}|^{2} d s\right]^{1 / 2} \\
& \cdot\left[\sum_{K \in \mathcal{T}_{h}}\left\|\nabla \mathbf{u}-\nabla_{h} \mathbf{w}\right\|_{L^{1}(\partial K)}^{2}+\|p-q\|_{L^{1}(\partial K)}^{2}\right]^{1 / 2} \\
\leq & C\|\mathbf{v}\|_{h}\left[\sum_{K \in \mathcal{T}_{h}}\left\|\nabla \mathbf{u}-\nabla_{h} \mathbf{w}\right\|_{L^{1}(\partial K)}^{2}+\|p-q\|_{L^{1}(\partial K)}^{2}\right]^{1 / 2} .
\end{aligned}
$$


In addition, the third assertion in Lemma 1 implies that

$$
\begin{aligned}
& \frac{\left|E_{h}(\mathbf{u}-\mathbf{w}, p-q ; \mathbf{v})\right|}{\|\mathbf{v}\|_{h}} \\
& \leq C\left[\sum_{K \in \mathcal{T}_{h}}\left[|\mathbf{u}-\mathbf{w}|_{H^{1}(K)}^{2}+\|p-q\|_{L^{2}(K)}^{2}\right]\right. \\
& \quad+\sum_{K \in \mathcal{K}_{\text {int }}} h_{K}^{2}\left[|\mathbf{u}-\mathbf{w}|_{H^{2}(K)}^{2}+|p-q|_{H^{1}(K)}^{2}\right] \\
& \left.\quad+\sum_{K \in \mathcal{K}_{\text {vert }}} h_{K}^{2-2 \beta_{K}}\left[|\mathbf{u}-\mathbf{w}|_{H_{\beta_{K}}^{2,2}(K)}^{2}+|p-q|_{H_{\beta_{K}}^{1,1}(K)}^{2}\right]\right]^{1 / 2} .
\end{aligned}
$$

Finally, applying (14) and using the fact $\nabla(q-\widetilde{q}) \equiv 0$ results in

$$
\begin{aligned}
& \frac{\left|E_{h}(\mathbf{u}-\mathbf{w}, p-q ; \mathbf{v})\right|}{\|\mathbf{v}\|_{h}} \\
& \leq C\left[E_{1}^{2}+\sum_{K \in \mathcal{K}_{\text {int }}} h_{K}^{2}\left[|\mathbf{u}-\mathbf{w}|_{H^{2}(K)}^{2}+|p-\widetilde{q}|_{H^{1}(K)}^{2}\right]\right. \\
& \left.\quad+\sum_{K \in \mathcal{K}_{\text {vert }}} h_{K}^{2-2 \beta_{K}}\left[|\mathbf{u}-\mathbf{w}|_{H_{\beta_{K}}^{2,2}(K)}^{2}+|p-\widetilde{q}|_{H_{\beta_{K}}^{1,1}(K)}^{2}\right]\right]^{1 / 2} \\
& \leq C\left(E_{1}+E_{2}+E_{3}\right),
\end{aligned}
$$

for all $\mathbf{v} \in \mathbf{V}_{h}$. Combining the bounds (13)-(15) with (12) completes the proof.

Remark 5 The factor $|\underline{k}|^{3}$ in the error bound of Theorem 2 is growing algebraically with the approximation orders. This is due to the decrease of the discrete inf-sup condition as $|\underline{k}|^{-1}$ and the definition of $\mathrm{c}$. Thus, the result in Theorem 2 leads to slightly suboptimal convergence rates in the pure $p$-version of the DGFEM; see also [29]. In the $h p$-version of the DGFEM, however, the error contributions $E_{1}, E_{2}$, and $E_{3}$ converge at exponential rates in the number of degrees of freedom, thereby absorbing the algebraic growth in $|\underline{k}|$.

\section{Exponential rates of convergence}

The aim of this section is to show that the error estimates in Theorem 2 are exponentially convergent on geometric meshes.

\subsection{Geometric meshes}

In order to resolve singular solution behavior near corners we introduce meshes that are geometrically refined towards the vertices of $\Omega$. First, we define the basic geometric meshes on $\hat{Q}=(0,1)^{2}$. 
Definition 2 Fix $n \in \mathbb{N}_{0}$ and $\sigma \in(0,1)$. On $\hat{Q}$, the geometric mesh $\Delta_{n, \sigma}$ with $n+1$ layers and grading factor $\sigma$ is created recursively as follows: If $n=0, \Delta_{0, \sigma}=\{\hat{Q}\}$. Given $\Delta_{n, \sigma}$ for $n \geq 0, \Delta_{n+1, \sigma}$ is generated by subdividing the square $K$ with $0 \in \bar{K}$ into four smaller rectangles by dividing its sides in $a \sigma:(1-\sigma)$ ratio.

An example of a basic geometric mesh is shown in Fig. 1. We denote the elements in the basic geometric mesh by $\left\{K_{i j}\right\}$ as indicated there. We say that the elements $K_{1 j}, K_{2 j}$ and $K_{3 j}$ constitute layer $j$ for $j \geq 2$ while $K_{11}$ is the element at the origin.

Definition 3 A geometric mesh $\mathcal{T}_{n, \sigma}$ in the polygon $\Omega \subset \mathbb{R}^{2}$ is obtained by mapping the basic geometric meshes $\Delta_{n, \sigma}$ from $\hat{Q}$ affinely to a vicinity of each convex corner of $\Omega$. At reentrant corners three suitably scaled copies of $\Delta_{n, \sigma}$ are used (as shown in Fig. 2). The remainder of $\Omega$ is subdivided with a fixed affine and quasi-uniform partition.

In Fig. 2 this local geometric refinement is illustrated. For ease of exposition, we consider only mesh patches that are identically refined with the same parameters $\sigma$ and $n$, although different grading factors and numbers of layers may be used for the partition of each corner patch.

Definition 4 A polynomial degree distribution $\underline{k}$ on a geometric mesh $\mathcal{T}_{n, \sigma}$ is called linear with slope $\mu>0$ if the elemental polynomial degrees are layerwise constant in the geometric patches and given by $k_{j}:=\max (2,\lfloor\mu j\rfloor)$ in layer $j, j=1, \ldots, n+1$. In the interior of the domain the elemental polynomial degree is set constant to $\max (2,\lfloor\mu(n+1)\rfloor)$.

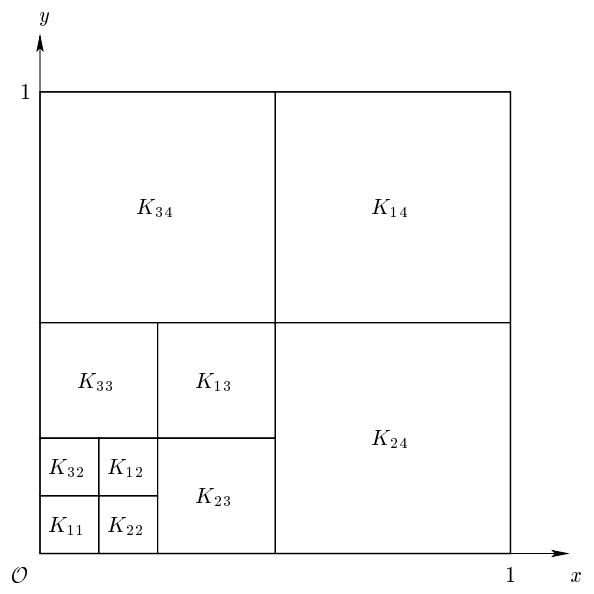

Fig. 1. The geometric mesh $\Delta_{n, \sigma}$ with $n=3$ and $\sigma=0.5$. The elements are numbered as indicated. 


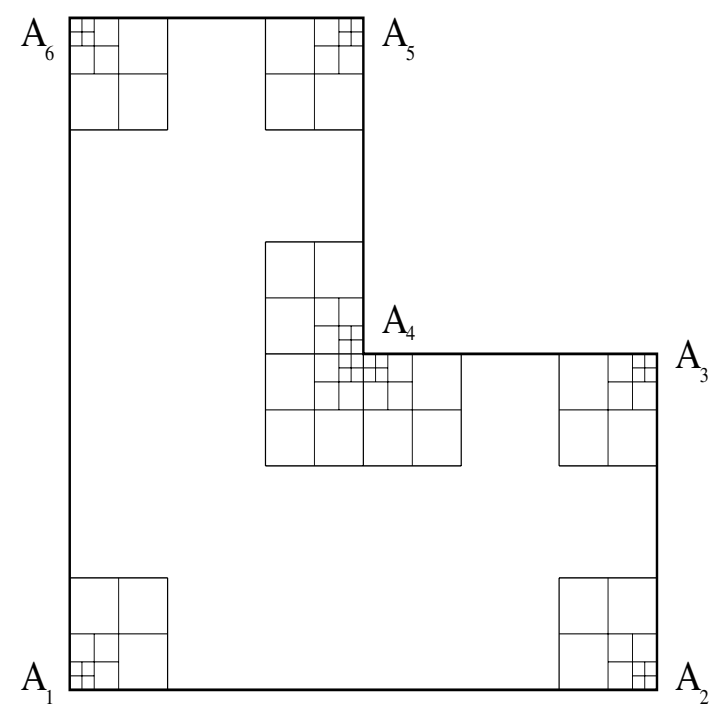

Fig. 2. Local geometric refinement near vertices $\left\{A_{i}\right\}$ of $\Omega$. At the reentrant corner $A_{4}$ three suitably scaled copies of $\Delta_{n, \sigma}$ are used. In all corners, $n=3$ and $\sigma=0.5$.

\subsection{Exponential convergence}

Our main result establishes exponential convergence of the mixed $h p$-DGFEM.

Theorem 3 Assume (3) and that the exact solution (u, p) of the Stokes system satisfies (4) with $\underline{\beta}_{\min }<\underline{\beta}<1$. Let $\left(\mathbf{u}_{h}, p_{h}\right) \in \mathbf{V}_{h} \times Q_{h}$ be the DGFEM approximation (8) on geometric meshes $\mathcal{T}_{n, \sigma}$. Then there exists $\mu_{0}=\mu_{0}(\sigma, \beta)>0$ such that for linear degree vectors $\underline{k}$ with slope $\mu \geq \mu_{0}$ there holds the error estimate

$$
\left\|\mathbf{u}-\mathbf{u}_{h}\right\|_{h}+\left\|p-p_{h}\right\|_{L^{2}(\Omega)} \leq C \exp \left(-b N^{1 / 3}\right)
$$

with constants $C, b>0$ independent of $N=\operatorname{dim}\left(\mathbf{V}_{h}\right) \approx \operatorname{dim}\left(Q_{h}\right)$.

Remark 6 If the polynomial degree is chosen to be constant throughout the mesh, i.e., $k_{K}=k$ for all $K \in \mathcal{T}_{h}$, exponential convergence is still obtained by choosing $k$ proportionally to the number $n$ of layers. This is due to the fact that the interpolant constructed for the proof of Theorem 3 still can be used for $k=\max (2,\lfloor\mu(n+1)\rfloor)$.

Proof. We proceed in two steps.

Step 1: We consider first the case where $\Omega=\hat{Q}$ and $\mathcal{T}_{n, \sigma}=\Delta_{n, \sigma}$ is the basic geometric mesh from Definition 2. From [28, Proposition 27] and [20] or [30, Lemma 4.25], there exist $\widetilde{q}_{11} \in \mathcal{Q}^{0}\left(K_{11}\right)$ and $\mathbf{w}_{11} \in \mathcal{Q}^{1}\left(K_{11}\right)^{2}$ such that 


$$
\begin{aligned}
\| p & -\widetilde{q}_{11} \|_{L^{2}\left(K_{11}\right)}^{2}+h_{K_{11}}^{2-2 \beta_{K_{11}}}\left|p-\widetilde{q}_{11}\right|_{H_{\beta_{K_{11}}}^{1,1}\left(K_{11}\right)}^{2} \\
& \leq C \sigma^{2 n\left(1-\beta_{K_{11}}\right)}|p|_{H_{\beta_{K_{11}}}^{1,1}\left(K_{11}\right)}^{2}
\end{aligned}
$$

and

$$
\begin{aligned}
& h_{K_{11}}^{-2}\left\|\mathbf{u}-\mathbf{w}_{11}\right\|_{L^{2}\left(K_{11}\right)}^{2}+\left|\mathbf{u}-\mathbf{w}_{11}\right|_{H^{1}\left(K_{11}\right)}^{2} \\
& \quad+h_{K_{11}}^{2-2 \beta_{K_{11}}}\left|\mathbf{u}-\mathbf{w}_{11}\right|_{H_{\beta_{K_{11}}}^{2,2}\left(K_{11}\right)}^{2} \leq C \sigma^{2 n\left(1-\beta_{K_{11}}\right)}|\mathbf{u}|_{H_{\beta_{K_{11}}}^{2,2}\left(K_{11}\right)}^{2} .
\end{aligned}
$$

Moreover, for $K_{i j} \in \mathcal{K}_{\text {int }}$ there are $\widetilde{q}_{i j} \in \mathcal{Q}^{k_{K_{i j}}-1}\left(K_{i j}\right)$ and $\mathbf{w}_{i j} \in \mathcal{Q}^{k_{K_{i j}}}\left(K_{i j}\right)^{2}$ such that

$$
\begin{aligned}
\| p & -\widetilde{q}_{i j} \|_{L^{2}\left(K_{i j}\right)}^{2}+h_{K_{i j}}^{2}\left|p-\widetilde{q}_{i j}\right|_{H^{1}\left(K_{i j}\right)}^{2} \\
& \leq C \sigma^{2(n+2-j)\left(1-\beta_{K_{11}}\right)} \frac{\Gamma\left(k_{K_{i j}}-s_{i j}+1\right)}{\Gamma\left(k_{K_{i j}}+s_{i j}-1\right)}\left(\frac{\rho}{2}\right)^{2 s_{i j}}\|p\|_{H_{\beta_{K_{11}}}^{s_{i j}+3,1}\left(K_{i j}\right)}^{2}
\end{aligned}
$$

and

$$
\begin{aligned}
& h_{K_{i j}}^{-2}\left\|\mathbf{u}-\mathbf{w}_{i j}\right\|_{L^{2}\left(K_{i j}\right)}^{2}+\left|\mathbf{u}-\mathbf{w}_{i j}\right|_{H^{1}\left(K_{i j}\right)}^{2}+h_{K_{i j}}^{2}\left|\mathbf{u}-\mathbf{w}_{i j}\right|_{H^{2}\left(K_{i j}\right)}^{2} \\
& \quad \leq C \sigma^{2(n+2-j)\left(1-\beta_{K_{11}}\right)} \frac{\Gamma\left(k_{K_{i j}}-s_{i j}+1\right)}{\Gamma\left(k_{K_{i j}}+s_{i j}-1\right)}\left(\frac{\rho}{2}\right)^{2 s_{i j}}\|\mathbf{u}\|_{H_{\beta_{K_{11}}}^{s_{i j}+3,2}\left(K_{i j}\right)}^{2}
\end{aligned}
$$

for any $1 \leq i \leq 3,2 \leq j \leq n+1$ and $s_{i j} \in\left[1, k_{K_{i j}}\right]$. Here, $\rho=$ $\max \left(1, \sigma^{-1}(1-\sigma)\right)$. This was proved, e.g., in [28, Sect. 5.2] in all details. Referring to Theorem 2 implies that

$$
\begin{aligned}
\| \mathbf{u} & -\mathbf{u}_{h}\left\|_{h}^{2}+\right\| p-p_{h} \|_{L^{2}(\Omega)}^{2} \leq C|\underline{k}|^{3} \sigma^{2 n\left(1-\beta_{K_{11}}\right)}\left[\Psi_{K_{11}}^{2,1}(\mathbf{u}, p)\right. \\
& \left.+\sum_{i=1}^{3} \sum_{j=2}^{n+1} \sigma^{2(2-j)\left(1-\beta_{K_{11}}\right)} \frac{\Gamma\left(k_{K_{i j}}-s_{i j}+1\right)}{\Gamma\left(k_{K_{i j}}+s_{i j}-1\right)}\left(\frac{\rho}{2}\right)^{2 s_{i j}} \Psi_{K_{i j}}^{s_{i j}+3, s_{i j}+3}(\mathbf{u}, p)\right],
\end{aligned}
$$

where

$$
\Psi_{K}^{m, l}(\mathbf{u}, p):=\|\mathbf{u}\|_{H_{\beta_{K_{11}}, 2}^{m, 2}(K)}^{2}+\|p\|_{H_{\beta_{K_{11}}}^{l, 1}(K)}^{2} .
$$

In [3,19] or [30, Sect. 4.5.3] it was shown that there exist $s_{i j}, 1 \leq i \leq 3,2 \leq$ $j \leq n+1$ and $\mu_{0}>0$ such that, for linear polynomial degree distributions as in Definition 4 with slope $\mu \geq \mu_{0}$, the right-hand side of (16) is exponentially small with respect to $N$. More precisely, there holds:

$$
\left\|\mathbf{u}-\mathbf{u}_{h}\right\|_{h}+\left\|p-p_{h}\right\|_{L^{2}(\hat{Q})} \leq C N^{3 / 4} \exp \left(-b N^{1 / 3}\right) \leq \widetilde{C} \exp \left(-\widetilde{b} N^{1 / 3}\right) .
$$


Here, we have also used the (pessimistic) bound $|\underline{k}|^{2} \leq N$ and have absorbed the algebraically growing term $N^{3 / 4}$ with the second term that decays exponentially fast with respect to $N$.

Step 2: Let now $\mathcal{T}_{n, \sigma}$ be a geometric mesh on the polygon $\Omega$, as in Definition 4 . We recall that $\mathcal{T}_{n, \sigma}$ is obtained by mapping affinely up to three geometric mesh patches $\Delta_{n, \sigma}$ to a neighborhood of each corner. On each of these patches, we can construct an interpolant $(\mathbf{w}, q)$ as in Step 1, remarking that a generalization of the result there to affinely mapped meshes can be established straightforwardly; see, e.g., [20,19,30] and the references cited therein.

This completes the proof.

\section{Conclusions}

In this paper, we have presented the first proof of exponential convergence for mixed $h p$-DGFEM for Stokes flow with piecewise analytic data on geometric meshes with linearly increasing approximation orders. The proof relies on a combination of new trace theorems for functions in weighted Sobolev spaces and standard $h p$-approximation techniques. We point out that the exponential convergence result proved in this work can be straightforwardly extended to mixed formulations of linear elasticity problems with nearly incompressible materials. The numerical validation of the $h p$-scheme proposed in this paper is the subject of ongoing work and will be presented elsewhere.

Acknowledgements. The authors are indebted to C. Schwab (ETH, Zürich) for many useful discussions and suggestions. This work has been initiated at the Oberwolfach Meeting "Discontinuous Galerkin Methods" during the week of 4/21-4/27/2002. The authors wish to acknowledge the excellent working conditions and stimulating atmosphere in the research institute of Oberwolfach.

\section{References}

1. Arnold, D.N., Brezzi, F., Cockburn, B., Marini, L.D.: Unified analysis of discontinuous Galerkin methods for elliptic problems. SIAM J. Numer. Anal. 39, 1749-1779 (2001)

2. Babuška, I., Guo, B.Q.: The $h p$-version of the finite element method for domains with curved boundaries. SIAM J. Numer. Anal. 25, 837-861 (1988)

3. Babuška, I., Guo, B.Q.: Regularity of the solution of elliptic problems with piecewise analytic data, I. SIAM J. Math. Anal. 19, 172-203 (1988)

4. Babuška, I., Guo, B.Q.: Regularity of the solution of elliptic problems with piecewise analytic data, II. SIAM J. Math. Anal. 20, 763-781 (1989)

5. Baker, G.A., Jureidini, W.N., Karakashian, O.A.: Piecewise solenoidal vector fields and the Stokes problem. SIAM J. Numer. Anal. 27, 1466-1485 (1990) 
6. Bernardi, C., Maday, Y.: Approximations Spectrales de Problèmes aux Limites Elliptiques. New York: Springer-Verlag, 1992

7. Bernardi, C., Maday, Y.: Uniform inf-sup conditions for the spectral discretization of the Stokes problem. Math. Models Meth. Appl. Sci. 9, 395-414 (1999)

8. Boillat, E., Stenberg, R.: An $h p$-error analysis of some Galerkin least squares methods for the elasticity equations. Technical Report 21-94, Helsinki University of Technology, 1994

9. Brezzi, F., Fortin, M.: Mixed and hybrid finite element methods. In: Springer Series in Computational Mathematics, volume 15. New York: Springer-Verlag, 1991

10. Cockburn, B.: Discontinuous Galerkin methods for convection-dominated problems. In T. Barth and H. Deconink, (eds), High-Order Methods for Computational Physics, volume 9 of Lect. Notes Comput. Sci. Engrg. New York: Springer-Verlag, 1999, pp. 69-224

11. Cockburn, B., Kanschat, G., Schötzau, D.: The local discontinuous Galerkin method for the Oseen equations. Math. Comp. to appear

12. Cockburn, B., Kanschat, G., Schötzau, D., Schwab, C.: Local discontinuous Galerkin methods for the Stokes system. SIAM J. Numer. Anal. 40, 319-343 (2002)

13. Cockburn, B., Karniadakis, G.E., Shu, C.-W.: The development of discontinuous Galerkin methods. In: B. Cockburn, G.E. Karniadakis, and C.-W. Shu, (eds), Discontinuous Galerkin Methods: Theory, Computation and Applications, volume 11, 2000, pp. $3-50$

14. Cockburn, B., Shu, C.-W.: Runge-Kutta discontinuous Galerkin methods for convection-dominated problems. J. Sci. Comput. 16, 173-261 (2001)

15. Dauge, M.: Stationary Stokes and Navier-Stokes systems on two- or three-dimensional domains with corners, I. Linearized equations. SIAM J. Math. Anal. 20, 74-97 (1989)

16. Franca, L., Stenberg, R.: Error analysis of some Galerkin least squares methods for the elasticity equations. SIAM J. Numer. Anal. 28, 1680-1697 (1991)

17. Girault, V., Raviart, P.A.: Finite Element Methods for Navier-Stokes Equations. New York: Springer-Verlag, 1986

18. Girault, V., Rivière, B., Wheeler, M.F.: A discontinuous Galerkin method with nonoverlapping domain decomposition for the Stokes and Navier-Stokes problems. Technical Report 02-08, TICAM, UT Austin, 2002

19. Guo, B.Q.: The $h p$-version of the finite element method for elliptic equations of order $2 m$. Numer. Math. 53, 199-224 (1988)

20. Guo, B.Q., Babuška, I.: The $h p$-version of the finite element method, I: The basic approximation results. Comp. Mech. 1, 21-41 (1986)

21. Guo, B.Q., Babuška, I.: The $h p$-version of the finite element method, II: General results and applications. Comp. Mech. 1, 203-226 (1986)

22. Guo, B.Q., Babuška, I.: On the regularity of elasticity problems with piecewise analytic data. Adv. Appl. Math. 14, 307-347 (1993)

23. Guo, B.Q., Schwab, C.: Analytic regularity of Stokes flow in polygonal domains. Technical Report 2000-18, SAM, ETH Zürich, 2000

24. Hansbo, P., Larson, M.G.: Discontinuous finite element methods for incompressible and nearly incompressible elasticity by use of Nitsche's method. Comput. Methods Appl. Mech. Engrg. 191, 1895-1908 (2002)

25. Karakashian, O.A., Jureidini, W.N.: A nonconforming finite element method for the stationary Navier-Stokes equations. SIAM J. Numer. Anal. 35, 93-120 (1998)

26. Orlt, M.: Regularity and FEM-Error Estimates for General Boundary Value Problems of the Navier-Stokes Equations. PhD thesis, Dept. of Math. Universität Stuttgart, 1998 
27. Quarteroni, A.: Some results of Bernstein and Jackson type for polynomial approximation in $L^{p}$ spaces. Japan J. Appl. Math. 1, 173-181 (1984)

28. Schötzau, D., Schwab, C.: Exponential convergence in a Galerkin least squares $h p$-FEM for Stokes flow. IMA J. Numer. Anal. 21, 53-80 (2001)

29. Schötzau, D., Schwab, C., Toselli, A.: Mixed $h p$-DGFEM for incompressible flows. SIAM J. Numer. Anal. 40, 2171-2194 (2003)

30. Schwab, C.: $p$ - and $h p$-FEM - Theory and Application to Solid and Fluid Mechanics. Oxford: Oxford University Press, 1998

31. Schwab, C., Suri, M.: Mixed $h p$-finite element methods for Stokes and NonNewtonian flow. Comput. Methods Appl. Mech. Engrg. 175, 217-241 (1999)

32. Stenberg, R., Suri, M.: Mixed $h p$-finite element methods for problems in elasticity and Stokes flow. Numer. Math. 72, 367-389 (1996)

33. Toselli, A.: $h p$-discontinuous Galerkin approximations for the Stokes problem. Math. Models Methods Appl. Sci. 12, 1565-1616 (2002)

34. Wihler, T.P.: Locking-free DGFEM for elasticity problems in polygons. IMA J. Numer. Anal. to appear

35. Wihler, T.P., Frauenfelder, P., Schwab, C.: Exponential convergence of the $h p$ DGFEM for diffusion problems. Comput. Math. Appl. to appear 